\title{
Scintigraphic Evidence for a Specific Long-Chain Fatty Acid Transporting System Deficit and the Genetic Background in a Patient With Hypertrophic Cardiomyopathy
}

\author{
Tomoaki Nakata, MD; Norifumi Nakahara, MD; Koichi Sohmiya, MD*; Fumio Okamoto, MD*; \\ Takao Tanaka, MD*; Keishiro Kawamura, MD*; Kazuaki Shimamoto, MD
}

\begin{abstract}
The mechanism of cardiac uptake of long-chain free fatty acids has not been fully determined. We encountered a hypertrophic cardiomyopathy patient who showed a lack of cardiac uptake of 2 different types of long-chain fatty acid analogues on the scintigraphic images. Flow cytometric analysis revealed no platelet or monocyte CD36 molecule expression (type I CD36 deficiency) and his CD36 gene showed homozygous mutation for ${ }^{478} \mathrm{C}$ to $\mathrm{T}$ substitution, leading to an abnormal CD36 amino acid sequence. These findings strongly suggest that a specific transporting system rather than a simple diffusion is commonly involved in the cardiac uptake of longchain free fatty acids in humans, and that the CD36 protein is the most likely candidate for the specific transporter and to explain scintigraphic defects on fatty acid imaging. (Jpn Circ J 1999; 63: 319-322)
\end{abstract}

Key Words: CD36; Fatty acid metabolism; Gene mutation; Hypertrophic cardiomyopathy; Scintigraphy

$\mathbf{T}$ he mechanism of cardiac uptake of long-chain free fatty acids remains controversial, although simple diffusion has hitherto been thought to be the mechanism! However, a specific transporting system of longchain fatty acids in myocardium has been suggested in the rat:,3 Recently, despite some unusual observations, several investigations have showed a lack of cardiac uptake of a synthesized radiolabeled long-chain free fatty acid analogue, iodine-123 (123I) labeled 15-(p-iodophenyl)-3R,S-methyl pentadecanoic acid (BMIPP), using a scintigraphic technique.-7 Although it is not clear whether this phenomenon is specific for cardiac BMIPP imaging or not, these findings suggest the presence of a specific protein involved in transporting long-chain free fatty acids. We encountered a patient with hypertrophic cardiomyopathy who showed a lack of cardiac uptakes of 2 different types of long-chain fatty acid analogues, ${ }^{123}$ I-labeled BMIPP and 123I labeled 15-(p-iodophenyl)-9-methylpentadecanoic acid (9MPA), and analyzed the genetic abnormality.

\section{Case Presentation}

A 43-year-old male with hypertrophic cardiomyopathy was admitted to the Sapporo Medical University Hospital for further evaluation of electrocardiographic abnormalities and exertional dyspnea, which had manifested 2 years before. He had no past or family history of coronary artery disease, heart failure, or sudden death, and suffered from

(Received October 14, 1998; revised manuscript received December 28, 1998; accepted January 5, 1999)

Second Department of Internal Medicine (Cardiovascular Medicine), Sapporo Medical University School of Medicine, Sapporo, *The Third Division, Department of Internal Medicine, Osaka Medical College, Takatsuki, Osaka, Japan

Mailing address: Tomoaki Nakata, MD, Second Department of Internal Medicine, Sapporo Medical University School of Medicine, S-1, W-16, Chuo-ku, Sapporo 060, Japan. E-mail: tnakata@ sapmed. ac.jp no systemic disorders such as endocrine diseases, hypertension, or renal failure. His rest electrocardiogram showed high voltages, left atrial overloading, and ST-segment depression in leads I, aVL, V5, and V6. Two-dimensional echocardiography revealed marked left ventricular hypertrophy of the interventricular septum, apex, and anterior wall with a wall-thickness of 17 to $20 \mathrm{~mm}$, and systolic anterior motion of mitral valve leaflets. His cardiac catheterization study revealed an intra-ventricular pressure gradient of $10 \mathrm{mmHg}$ at baseline, which increased to 120 $\mathrm{mmHg}$ during continuous intravenous infusion of isoproterenol hydrochloride $\left(0.02 \mu \mathrm{g} \mathrm{Kg}^{-1} \mathrm{~min}^{-1}\right)$. However, no other abnormality was detected, eg, in coronary anatomy and systolic function. From these findings, a diagnosis of an obstructive form of hypertrophic cardiomyopathy was made.

A 50-year-old male with severe aortic stenosis admitted for further evaluation of syncope was also studied for comparison with our hypertrophic cardiomyopathy patient. His 2-dimensional echocardiography and cardiac catheterization study revealed marked left ventricle hypertrophy (wall-thickness of 13 to $17 \mathrm{~mm}$ ), severely limited aortic valve opening due to severe adhesions and sclerosis, and an increased pressure gradient $(108 \mathrm{mmHg})$ between the left ventricle and aorta, but no other cardiac disorder was detected.

\section{Scintigraphic Imaging}

Cardiac fatty acid imaging was performed using 2 different iodinated long-chain fatty acid analogues, ${ }^{123}$ I-BMIPP and ${ }^{123} \mathrm{I}-9 \mathrm{MPA}$, as well as thallium perfusion imaging. Thallium-201 $\left({ }^{201} \mathrm{Tl}\right)(111 \mathrm{MBq}),{ }^{123}$ I-BMIPP $(111 \mathrm{MBq})$ (provided by Nihon Mediphysics, Ltd, Osaka, Japan) and 123I-9MPA (provided by Daiichi Radioisotope Laboratory, Ltd, Tokyo, Japan) were injected intravenously at resting and over-night fasting conditions on separate days. Ten min after the injections, planar thallium and 9MPA imagings were performed, and cardiac BMIPP imaging was 


\section{Thallium-201} 123| BMIPP 123| 9MPA
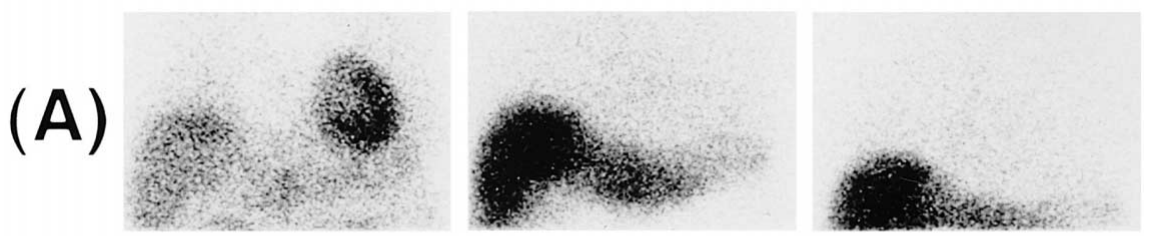

Fig 1. Planar images of thallium-201, 123I-BMIPP, and 123I-9MPA in the index patient with hypertrophic cardiomyopathy (A) and in a 50-year-old male with left ventricular hypertrophy due to aortic stenosis (B). The thallium images in both cases show increased cardiac activities due to left ventricular hypertrophy. The BMIPP and 9MPA scans (B, right 2 panels) also show similar findings in the aortic stenosis patient; in contrast, no cardiac uptake of BMIPP or 9MPA can be identified in the patient with hypertrophic cardiomyopathy (A, right 2 panels).
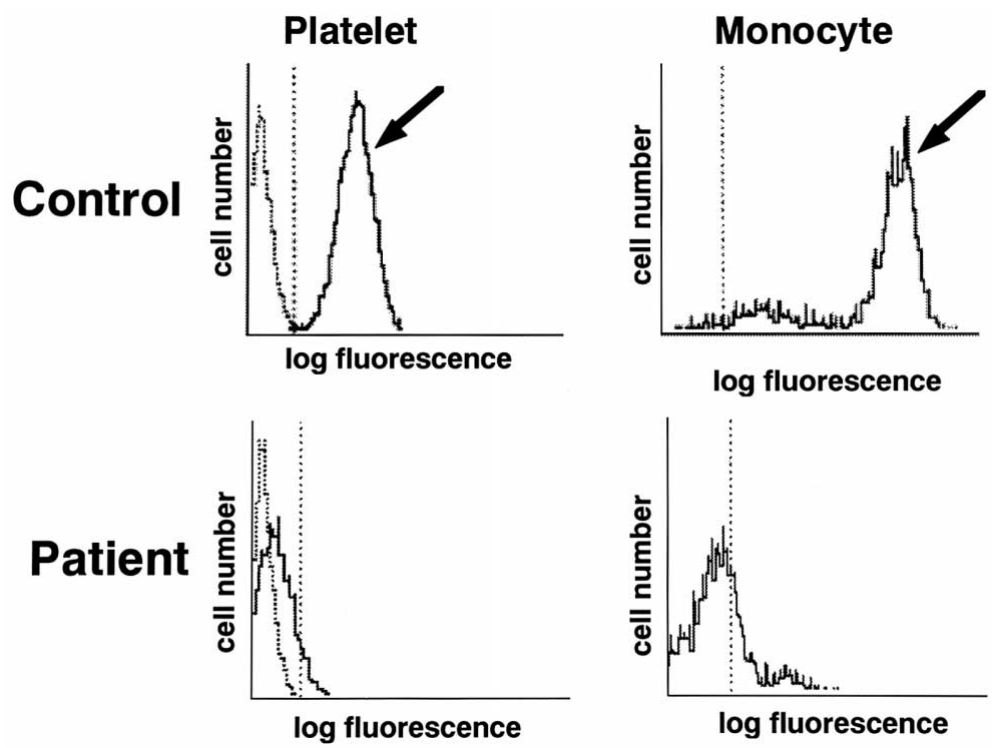

Fig 2. Flow cytometry analysis of CD36 molecule
expression on platelets (left panels) and on monocytes
(right panels). Compared to normal expressions
(arrows) of CD36 molecule both on platelets and on
monocytes in a control subject (upper panels), no
CD36 expression is observed on platelets or on mono-
cytes in this case (lower panels).

done $30 \mathrm{~min}$ after the tracer injection. These planar imagings were performed from an anterior view to acquire more than 500k counts. Subsequently, tomographic data were collected at 6-degree increments for $30 \mathrm{~s}$ per increment during a 360-degree rotation with a 3-head gamma camera (Toshiba GCA9300A/DI, Tokyo, Japan) equipped with a high-resolution parallel-hole collimator. Data acquisition was set for each energy window; the $159 \mathrm{keV}$ photopeak of ${ }^{123} \mathrm{I}$ with a $20 \%$ window and the $75 \mathrm{keV}$ photopeak of ${ }^{201} \mathrm{Tl}$ with a $10 \%$ window and the information was stored in a $128 \times 128$ word matrix nuclear medicine computer system. After serial transverse tomograms were reconstructed by a filtered backprojection algorithm with Shepp and Logan's filter, short-axis, vertical long-axis, and horizontal longaxis tomograms were obtained. Despite the increased thallium activity due to cardiac hypertrophy, no cardiac activity of BMIPP or 9MPA was identified in the index case (Fig 1A); on the contrary, comparable cardiac uptakes of thallium, BMIPP, and 9MPA were observed in the 50-yearold man with aortic stenosis and severe cardiac hypertrophy (Fig 1B).

\section{Flow Cytometry of the CD36 Molecule}

CD36 molecular expression on platelets and monocytes, which is a candidate of a specific transporter protein of long-chain fatty acids, 2,3,8,9 was measured by flow cytometry using anti-human CD36 monoclonal antibody as described previously? Briefly, following preparation by differential centrifugation at $250 \times \mathrm{g}$ for $10 \mathrm{~min}$ at room temperature, platelets and mononuclide cells were diluted to $10^{8} / \mathrm{ml}$ with phosphate buffered saline (PBS), respectively. After incubation for $30 \mathrm{~min}$ at $4^{\circ} \mathrm{C}$ with $10 \mu \mathrm{l}$ of a fluorescein isothiocyanate-labeled anti-CD 36 monoclonal antibody (OKM5; Ortho Diagnostic Systems, Raritan, NJ) and washing, flow cytometric analysis was performed using a CYTRON flow cytometer (Ortho) with an argon laser $(488 \mathrm{~nm})$. Fig 2 shows the results from a control subject and the present case. Compared to the appearance of CD36 molecules on both platelets and monocytes in the control, no definite expression was identified on platelets or monocytes in the index case (type I CD36 deficiency) ${ }^{10}$

\section{CD36 Gene Analysis}

The CD36 gene was analyzed using DNA isolated from peripheral blood samples with the QIAamp Blood Kit (QIAGEN) around the previously identified mutations: exon $4,{ }^{478} \mathrm{C}$ to $\mathrm{T}$ substitution; ${ }^{11}$ exon 5 , dinucleotide deletion of ${ }^{539} \mathrm{AC} ; 12$ and exon 10 , single nucleotide insertion of ${ }^{1159} \mathrm{~A} \cdot{ }^{13}$ After amplification of the interested regions by polymerase chain reaction (PCR), mutations were 


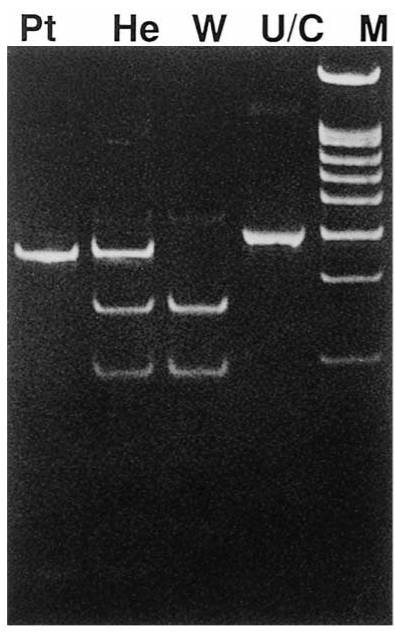

\section{${ }^{478} \mathrm{C} \rightarrow \mathrm{T}$ substitution}
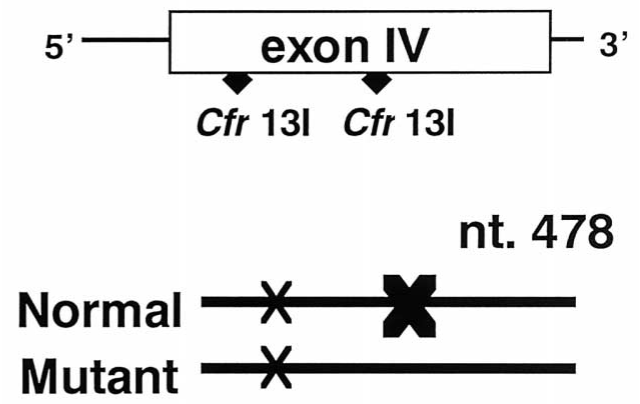

Fig 3. CD36 gene analysis demonstrating homozygous mutation for ${ }^{478} \mathrm{C}$ to $\mathrm{T}$ substitution (left panel). He, heterozygote; M, molecular marker (PBR322 digested with Hinf I); Pt, patient (homozygote); U/C, uncut fragment; and $\mathrm{W}$, digested wild type.

confirmed by endonuclease digestion using $C f r$ 13I, Ssp I, and $\mathrm{Xmn}$ I for ${ }^{478} \mathrm{C}$ to $\mathrm{T}$ substitution, dinucleotide deletion, and single nucleotide insertion, respectively. CD36 gene was also analyzed in these 3 exons by PCR-dependent preferential homoduplex formation assay. ${ }^{14}$ Compared to the gene expression in a wild-type genotype of CD36, the present patient showed homozygous mutation for ${ }^{478} \mathrm{C}$ to $\mathrm{T}$ substitution of CD36 gene (Fig 3).

\section{Discussion}

The present findings in the cardiomyopathy patient clearly demonstrated a lack of long-chain free fatty acid uptake in the myocardium by using 2 different types of fatty acid analogues synthesized for cardiac imaging, despite normal myocardial perfusion and normal liver uptake of both tracers. This phenomenon cannot be explained by a simple diffusion mechanism of long-chain fatty acid transport via sarcolemma. Therefore, a specific transporting system is possibly involved in cardiac uptake of long-chain fatty acids in human myocardium as well as in rat myocardium? There have been several studies reporting a lack of myocardial BMIPP by scintigraphic imaging4-7 However, it has not been determined whether this phenomenon can be seen in cardiac imaging with another fatty acid single photon emission computed tomography (SPECT) tracer. In addition to a cardiac BMIPP defect, a global deficit of myocardial uptake of ${ }^{123}$ I-9MPA, which is a relatively new compound for cardiac fatty acid imaging, 15 was observed in the present case, strongly suggesting the presence of a common system of long-chain fatty acid uptake in human myocardium. Positron emission computed tomography with carbon-11-labeled palmitate ${ }^{7}$ has showed that myocardium with a complete defect of BMIPP uptake also had a markedly reduced palmitate uptake. These findings also strongly suggested a common pathway of cardiac transport of long-chain fatty acids via sarcolemma. A defect of CD36 molecule expression on both platelets and monocytes is known as type I deficiency 10 It is revealed that CD36 protein is identical to cardiac long-chain fatty acid transporter (C-FAT) identified in rat and bovine myocardium 3,8 The present case had a CD36 gene deficit due to homozygous mutation for ${ }^{478} \mathrm{C}$ to $\mathrm{T}$ substitution, which results in the replacement of proline ( ${ }^{90}$ Pro) by serine (Ser) in the CD36 amino acid sequence which may provide some post-translational processing failure. This type of mutation is one of the CD36 mutations responsible for impaired CD36 expression on blood cells. ${ }^{1-13}$ Therefore, it is likely that type I CD36 deficiency involves a deficit of CD36 expression on myocytes as well as on platelets and monocytes, and is responsible for impaired uptake of long-chain fatty acids and their analogues, BMIPP and 9MPA, in the myocardium. In clinical trial phases I, II, and III for cardiac imaging with ${ }^{123}$ IBMIPP performed from 1990 to 1992 in Japan, poor cardiac uptake of the radiotracer was documented in 4 $(0.54 \%)$ out of 746 subjects (unpublished data provided by Nihon Mediphysics, Ltd, Osaka, Japan, 1998). Kudoh et al ${ }^{7}$ observed a complete lack of myocardial 123I-BMIPP uptake in 11 of 1258 patients (approximately $0.9 \%$ ); 2 of the 11 patients had hypertrophic cardiomyopathy, and the prevalence of zero cardiac uptake in 113 patients with hypertrophic cardiomyopathy was $1.8 \%$. However, CD36 expression was not investigated in this study. The prevalence of abnormal CD36 expression was approximately $3 \%$ in healthy Japanese blood donors and $0.34 \%$ in theUnited States, ${ }^{17}$ and the prevalence of type I CD36 deficiency is much lower at approximately one-tenth of that of type II CD36 deficiency. However, the presence or absence of a cardiac defect of long-chain fatty acid (BMIPP or 9-MPA) uptake in these healthy subjects showing CD36 deficiency is unclear. Although CD36 expression was not investigated in the aortic stenosis patient who showed cardiac hypertrophy but normal uptakes of BMIPP and 9MPA, previous reports ${ }^{5,16}$ have demonstrated that all patients showing a global BMIPP defect have type I CD36 deficiency. Our recent observations (unpublished data) showed that all of 4 hypertrophic cardiomyopathy patients showing no cardiac BMIPP uptake were type I CD36 deficiency and that 12 (29\%) of 41 hypertrophic cardiomyopathy patients who showed regional BMIPP defect were type II CD36 deficiency, indicating a close correlation between CD36 molecule expression and impaired cardiac uptake of long-chain free fatty acids.

Cardiac fatty acid metabolism is a major energy production system in aerobic conditions in the myocardium ${ }^{1}$ and cardiac BMIPP activity is closely related to intra-cellular ATP content 18 Nevertheless, the pathophysiological implications of type I CD36 deficiency or complete deficit of myocardial uptake of long-chain free fatty acids have not been fully determined. In the present case, there was no specific abnormality in clinical or functional states rather 
than those explained by hypertrophic cardiomyopathy itself. Positron imaging with ${ }^{18} \mathrm{~F}$-deoxyglucose showed a substrate shift from long-chain free fatty acids to glucose in patients with cardiac BMIPP defect6,7 Therefore, cardiac metabolisms using medium-chain and short-chain fatty acids, glucose, amino acids, and lactate may be augmented to compensate the energy production system in response to reduced (or, originally low) utilization of long-chain fatty acids in the myocardium. On the other hand, a CD36 abnormality may be associated with some form of cardiac hypertrophy, $5,16,19$ Two previous reports 5,16 demonstrated a correlation between impaired cardiac BMIPP uptake and CD36 expression deficiency in 11 patients with hypertrophic cardiomyopathy; 3 patients with type I CD36 deficiency showed no cardiac uptake of BMIPP and 4 out of 8 patients with type II CD36 deficiency showed reduced BMIPP uptake. In these studies, however, CD36-related genes were not investigated5,16 Kusaka and Tanaka et al ${ }^{19}$ reported induction of cardiac hypertrophy in rats by inhibition of CD36 molecule by sulfo-N-succinimidyl palmitate. Although the mechanism behind cardiac hypertrophy due to CD36 inhibition in sarcolemma is undetermined, increased carbohydrate utilization in response to reduced utilization of long-chain fatty acids ${ }^{6-8,20}$ might be related to signal transduction of the myosin heavy chain gene 21,22 and might activate growth factor (TGFa, bFGF) production? 23 Further investigations are, however, necessary to determine the etiological implications of impaired CD36 molecule expression and its genetic deficits in human hypertrophic cardiomyopathy.

\section{Conclusions}

Cardiac defects of BMIPP and 9MPA uptake on scintigraphic imagings strongly suggest that myocardial uptake of long-chain free fatty acids commonly depends upon a sarcolemmal-specific transporting system in humans. A lack of CD36 protein expression and the genetic abnormality, homozygous mutation for ${ }^{478} \mathrm{C}$ to $\mathrm{T}$ substitution, is a likely mechanism to explain the cardiac defect of uptake of long-chain fatty acids analogues. The present findings suggest a need to investigate in more detail the mechanism of transporting system of long-chain free fatty acids in the myocardium, the genetic background, and the pathophysiological implications in human cardiac hypertrophy.

\section{Acknowledgments}

The authors would like to thank the Nuclear Medicine staff at Sapporo Medical University Hospital, Sapporo, Japan, for their technical assistance. We are also grateful to Dr Naomi M. Anderson, Calgary for editorial assistance.

\section{References}

1. Katz AM: Oxidative metabolism. In: Katz AM, editor. Physiology of the heart. New York: Raven Press, 1992: 98-104

2. Stremmel W: Fatty acid uptake by isolated rat heart myocytes represents a carrier mediated transport process. J Clin Invest 1988; 81: $844-852$

3. Abumrad N, El-Maghrabi M, Amri E-Z, Lopez E, Grimaldi P: Cloning of a rat adipocyte membrane protein implicated in binding or transport of long-chain fatty acids that is induced during preadipocyte differentiation. J Biol Chem 1993; 268: 17665-17668
4. Hwang EH, Yamashita A, Takemori H, Taki J, Nakajima K, Bunko $\mathrm{H}$, et al: Absence of myocardial I-123 BMIPP uptake in a family. Ann Nucl Med 1996; 10: 445-448

5. Watanabe K, Toba K, Ogawa Y, Aizawa Y, Tanabe N, Miyajima S, et al: Different patterns of ${ }^{123}$ I-BMIPP myocardial accumulation in patients with type I and II CD36 deficiency. Kaku Igaku (Jpn J Nucl Med) 1997; 34: 1125-1230

6. Inoue $\mathrm{F}$, Hashimoto $\mathrm{T}$, Nishida $\mathrm{Y}$, Dohi $\mathrm{K}$, Matsushima A, Sakakibara H, et al: Absence of myocardial ${ }^{123}$ I-BMIPP uptake in the presence of a normal coronary angiogram and normokinetics on a left ventriculogram. Jpn Circ J 1997; 61: 263-267

7. Kudoh T, Tamaki N, Magata Y, Konishi J, Nohara R, Iwasaki A, et al: Metabolism substrate with negative myocardial uptake of iodine123-BMIPP. J Nucl Med 1997; 38: 548-553

8. Tanaka T, Kawamura K: Isolation of myocardial membrane longchain fatty acid-binding protein: Homology with a rat membrane protein implicated in the binding or transport of long-chain fatty acids. J Mol Cell Cardiol 1995; 27: 1613-1622

9. Tanaka T, Okamoto F, Sohmiya K, Kawamura K: Lack of myocardial iodine-123 15-(p-iodophenyl)-3-R,S-methylpentadecanoic acid (BMIPP) uptake and CD36 abnormality - CD36 deficiency and hypertrophic cardiomyopathy —. Jpn Circ J 1997; 61: 724-725

10. Yamamoto N, Akamatsu N, Sakuraba H, Yamazaki H, Tanoue K: Platelet glycoprotein IV (CD36) deficiency is associated with the absence (type I) or the presence (type II) of glycoprotein IV on monocytes. Blood 1994; 83: 392-397

11. Kashiwagi H, Tomiyama Y, Honda S, Kosugi S, Shiraga M, Nagao $\mathrm{N}$, et al: Molecular basis of CD36 deficiency: evidence that a ${ }^{478} \mathrm{C}$ $>$ T substitution (proline90 ->serine) in CD36 cDNA accounts for CD36 deficiency. J Clin Invest 1995; 95: 1040-1046

12. Kashiwagi H, Tomiyama Y, Kosugi S, Shiraga M, Lipsky RH, Kanayama Y, et al: Identification of molecular defects in a subject with type I CD36 deficiency. Blood 1994; 83: 3545-3552

13. Kashiwagi H, Tomiyama Y, Nozaki S, Honda S, Kosugi S, Shiraga $\mathrm{M}$, et al: A single nucleotide in codon 317 of the CD36 gene leads to CD36 deficiency. Arterioscler Thromb Vasc Biol 1996; 16: $1026-$ 1032

14. Oka T, Nakano H, Yamane A: Detection of mutations in the CFTR gene using the PCR-dependent referential homoduplex formation assay (PCR-PHFA) system. Nippon Rinsho 1996; 54: 518-524

15. Chouraqui P, Maddahi J, Henkin R, Karesh SM, Galie E, Berman DS: Comparison of myocardial imaging with iodine-123iodophenyl-9-methyl pentadecanoic acid and thallium-201-chloride for assessment of patients with exercise-induced myocardial ischemia. J Nucl Med 1991; 32: 447-452

16. Tanaka T, Sohmiya K, Kawamura K: Is CD36 deficiency an etiology of hereditary hypertrophic cardiomyopathy? J Mol Cell Cardiol 1997; 29: $121-127$

17. Yamamoto N, Ikeda H, Tandon NN, Herman J, Tomiyama Y, Mitani $\mathrm{T}$, et al: A platelet membrane glycoprotein (GP) deficiency in healthy blood donors: Nak ${ }^{\mathrm{a}-}$ platelet lack detectable GPIV (CD36). Blood 1990; 76: $1698-1703$

18. Fujibayashi Y, Yonekura Y, Takemura Y, Wada K, Matsumoto K, Tamaki N, et al: Myocardial accumulation of iodinated beta-methylbranched fatty acid analogue, iodine-125-15-(p-iodophenyl)-3(R,S) methyl pentadecanoic acid (BMIPP), in relation to ATP concentration. J Nucl Med 1990; 31: 1818-1822

19. Kusaka Y, Tanaka T, Okamoto F, Sohmiya K, Kawamura K: Effect of sulfo-N-succinimidyl palmitate on the rat heart: Myocardial longchain fatty acid uptake and cardiac hypertrophy. J Mol Cell Cardiol 1995; 27: $1605-1612$

20. Neely JR, Morgan HE: Relationship between carbohydrate and lipid metabolism and the energy balance of heart muscle. Annu Rev Physiol 1974; 36: 413-457

21. Rupp H, Elimban V, Dhalla NS: Sucrose feeding prevents changes in myosin isoenzymes and sarcoplasmic reticulum $\mathrm{Ca}^{2+}$-pump ATPase in pressure-loaded rat heart. Biochem Biophys Res Comm 1988; 156: 917-923

22. Rupp H, Jacob R, Dhalla NS: Signal transduction in myocardial hypertrophy and myosin expression. In: Korecky B, Dhalla NS, editors. Subcellular Basis of Contractile Failure. Boston: Kluwer Academic Publishers, 1990: 135-154

23. McClain DA, Paterson AJ, Roos MD, Wei X, Kudlow JE: Glucose and glucosamine regulate growth factor expression in vascular smooth muscle cells. Proc Natl Acad Sci USA 1992; 89: 917-923 